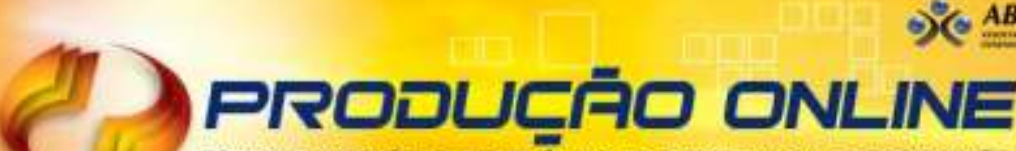 REVISTA CIENTIFICA ELETRONICA DE ENGENHARIA DE PRODUCAO ISSN 1676-1901
}

\section{DESENVOLVIMENTO DE MODELO DE BALANCED SCORECARD PARA INSTITUIÇÕES PRIVADAS DE ENSINO FUNDAMENTAL E MÉDIO ${ }^{1}$}

\section{DEVELOPMENT OF BALANCED SCORECARD MODEL FOR PRIVATE INSTITUTIONS OF ELEMENTARY AND SECUNDARY EDUCATION}

\author{
Izaac Shallon Gomes Costa* E-mail: ishallon@gmail.com \\ José Alfredo Ferreira Costa* E-mail: jafcosta@gmail.com \\ Kleber Cavalcanti Nobrega** E-mail: kleber@klebernobrega.com.br \\ * Universidade Federal do Rio Grande do Norte (UFRN), Natal, RN \\ ${ }^{*}$ Universidade Potiguar (UNP), Natal, RN
}

\begin{abstract}
Resumo: O Balanced Scorecard (BSC) é uma metodologia para gestão estratégica já consolidada mundialmente desde os anos 1990. Este sistema tem sido aplicado em empresas de diversos setores do mercado, em instituições privadas e públicas, devido à sua flexibilidade. O setor educacional está cada vez mais competitivo e há crescente busca por qualidade e melhoria da gestão das instituições de ensino. Diversos estudos mostram o sucesso do BSC em educação em diferentes países, mas poucos no âmbito da educação básica. Neste sentido, o objetivo deste trabalho foi o desenvolvimento de um modelo de BSC na área educacional, aplicável a escolas privadas que trabalham com ensino fundamental e nível médio. O modelo preliminar de objetivos e indicadores foi desenvolvido com base na literatura. A partir deste modelo inicial seguiu-se a elaboração de questionários, aplicados em entrevistas com gestores escolares, para realizar um estudo baseado no BSC, coletando informações para a escolha do conjunto de objetivos estratégicos para as quatro perspectivas do modelo. Foi utilizada a técnica chamada "bag of stars" na aplicação dos questionários. Os resultados alcançados foram: descrição da gestão estratégica nas escolas pesquisadas; o modelo de BSC para instituições privadas de ensino fundamental e médio; um mapa estratégico com as relações entre os objetivos.
\end{abstract}

Palavras-chave: Balanced Scorecard. Gestão Escolar. Estratégia. Mapa Estratégico. Bag of Stars.

Abstract: The Balanced Scorecard (BSC) is a methodology for strategic management worldwide consolidated since the 1990s. This system has been applied in various market sectors, in private and public institutions, because of its flexibility. The educational sector is increasingly competitive and there is growing demand for quality and the consequent need to improve the management of educational institutions. Several studies show the success of the BSC on education in different countries, but few in the context of basic education. Within this scope, the objective of this work is the development of a BSC model in education field, applicable to private schools that work with both elementary and middle level. The preliminary model of objectives and indicators for BSC was developed based on the literature review. From this initial model was followed by the development of questionnaires, that they were applied in interviews with school managers, to conduct a study based on BSC, collecting information for choosing the set of strategic objectives for the four perspectives of the model. A technique called "bag of stars" was used in the application of questionnaires. The results were: the description of the strategic management in the surveyed schools; the model of BSC for

1 O presente artigo é resultado de dissertação de mestrado defendida no programa de pósgraduação em Engenharia de Produção da UFRN (PEP-UFRN). O texto é inédito e não há nenhum tipo de conflito de interesses por parte dos autores 
elementary and high school private institutions; strategic map with the relationships between objectives.

Keywords: Balanced Scorecard. School Management. Strategic. Strategic Map. Bag of Stars.

\section{INTRODUÇÃO}

As organizações têm a necessidade de melhorar cada vez mais a qualidade de suas gestões, independentemente da área de atuação. Em mercados que se tornam mais competitivos com o passar do tempo, é preciso buscar informações sobre o desempenho das empresas e também sobre os resultados dos seus direcionamentos estratégicos.

Nesse contexto, uma ferramenta para gestão reconhecida mundialmente é o Balanced Scorecard (BSC), desenvolvido por Kaplan e Norton nos anos 90 e criado inicialmente como um sistema de medição de desempenho para empresas, mas que ao longo dos anos passou a ser usado como um sistema direcionador de gestão estratégica. O BSC é aplicável tanto em empresas privadas, foco original do modelo, como na esfera pública, onde sua utilização tem aumentado nos últimos anos (QUESADO et al., 2014; LIANG, 2012).

O setor da educação está inserido dentro dessas necessidades de medição de desempenho e gestão estratégica, e conforme apontado nesta pesquisa, há exemplos de sucesso de implementação e uso do BSC para escolas e universidades de diversos níveis e dimensões, de diferentes tipos de modalidade de ensino, tratando-se tanto de instituições públicas como privadas. Algumas instituições de ensino têm visto no BSC um modelo com o qual pode-se enfrentar desafios como a concorrência do mercado, o controle dos gastos e a prestação de contas através de um foco no atendimento ao cliente, questionando como oferecer maior valor aos seus alunos, e como melhorar seus processos contendo e reduzindo custos (REID, 2011; HLADCHENKO, 2015; DORWEILER; YAKHOU, 2005).

Assim, este trabalho traz uma pesquisa sobre o uso do BSC na área educacional, como ferramenta para gestão escolar com foco na esfera privada, valendo-se para isso de pesquisas relacionadas ao assunto nas principais bases de dados internacionais. Através da coleta e análise de artigos sobre BSC aplicado à educação, desenvolveu-se um modelo preliminar de objetivos e indicadores de desempenho a partir da literatura.

Revista Produção Online. Florianópolis, SC, v. 18, n. 4, p. 1181-1205, 2018. 
A partir do modelo obtido, elaborou-se um questionário para aplicação em escolas da região metropolitana do município do Natal, no estado do Rio Grande do Norte. A aplicação dos questionários foi realizada através de entrevistas, para validar o modelo preliminar, filtrando-se os principais objetivos e indicadores de cada perspectiva, de acordo com as informações obtidas através dos entrevistados, chegando-se assim a um modelo final. Para isso é utilizada a técnica "bag of stars". Essa técnica tem como um de seus principais fundamentos dar aos participantes uma quantidade limitada de fichas (estrelas) para escolher as melhores ideias dentro de um conjunto maior de opções. Dessa forma, a ideia é promover um filtro dentre as opções disponíveis num determinado processo de escolha (GARCIA; KLEIN, 2012).

Os objetivos do trabalho são: traçar um panorama da gestão das escolas participantes; desenvolver um modelo de BSC com objetivos e indicadores de desempenho para gestão escolar na esfera privada, com ênfase em instituições de ensino fundamental e médio; elaborar um mapa estratégico a partir do referido modelo.

\section{REVISÃO DE LITERATURA}

A revisão de literatura foi realizada através de pesquisas sobre trabalhos relacionados ao uso do BSC em instituições de ensino tanto de nível superior, como de nível médio e fundamental.

\subsection{BSC em universidades}

Esta seção mostra a análise de trabalhos relacionados à aplicação do BSC em Instituições de Ensino Superior (IES). Apesar do presente trabalho focar em instituições de ensino fundamental e médio, as pesquisas realizadas constataram a predominância de aplicações do BSC, no que tange à área educacional, primordialmente em universidades, e estes trabalhos também foram usados como base para o quadro preliminar composto nesta pesquisa, sendo, portanto, importantes para a análise. 
Neste sentido, Sayed (2013) apresenta como um dos seus maiores desafios a adequação do BSC para a realidade de diferentes IES. O estudo compreende o uso do BSC em trinta universidades ao redor do mundo, mostrando, inclusive, em muitos casos como elas optaram por modificar as perspectivas originais do BSC para atender a seus objetivos específicos.

Para identificar as universidades que adotaram o BSC, Sayed (2013) se baseou em informações disponíveis em sites das instituições, revistas e outras publicações. Portanto o número real pode ser maior. Mas, de acordo com o levantamento, a aplicação do BSC não é generalizada. As universidades pesquisadas em geral começaram a usar o BSC entre 2000 e 2005, não havendo muitas adesões ao sistema desde então. $O$ trabalho mostrou a individualidade do uso do BSC para cada caso e como o sistema é versátil.

Outro resultado interessante desenvolvido por Sayed (2013) foi a confecção de um modelo genérico de indicadores de desempenho para as universidades, ainda que o mesmo não tenha um potencial de sucesso claro. Esse modelo foi elaborado a partir da análise de aproximadamente 450 indicadores considerados pelas universidades, a maioria dos quais mostraram uma sobreposição considerável e foram analisados, recodificados, e categorizados com base nos fatores comuns incluídos nas perspectivas tradicionais do BSC. Sayed (2013) destaca em suas conclusões que indicadores de desempenho podem ser ferramentas poderosas para a avaliação estratégica nas universidades.

Respaldando esse pensamento, Schobel e Scholey (2012) afirmam que, uma vez corretamente implementado, o BSC é uma ferramenta de gerenciamento válida em instituições de ensino. O trabalho de Schobel e Scholey (2012) mostra a aplicação do BSC em universidades. O objetivo foi mostrar o uso do BSC em um ambiente de aprendizagem à distância de educação superior, destacando a importância de estratégias financeiras, fundamental num momento de crise financeira surgido durante a implementação do trabalho. Os autores mostram o desenvolvimento do BSC na universidade onde lecionam, incluindo um scorecard e um mapa estratégico, que identificou os stakeholders, os processos de negócios, a renovação e as estratégias financeiras. Eles confrontaram opiniões divergentes de autores sobre o uso do BSC na área educacional, refutando afirmações contrárias à 
prática com exemplos de sucesso da aplicabilidade do modelo no ambiente educacional.

Em publicação um pouco mais antiga, de 2005, Dorweiler e Yakhou tiveram como objetivo proporcionar um quadro para um scorecard relativo ao desempenho de administradores acadêmicos. No artigo, os autores afirmam que a literatura mostra que as organizações empresariais, bem como instituições acadêmicas, precisam repensar suas estratégias e operações por causa das mudanças de ambiente. O BSC é descrito como uma nova abordagem para enfrentar estes desafios. O mesmo foi mostrado como uma ferramenta eficaz para avaliar uma organização e seu desempenho (DORWEILER; YAKHOU, 2005).

Como limitações da pesquisa, embora o estudo forneça um quadro geral de um BSC para instituições acadêmicas, ele não traz uma lista exaustiva dos objetivos acadêmicos e medidas associadas para avaliação. Como implicações práticas, ele traz uma orientação para os administradores acadêmicos em sua busca por maneiras de melhorar a eficácia e demonstrar a responsabilidade para o governo e o público. O estudo oferece insights sobre como traduzir a base de negócios do BSC para o ambiente acadêmico (DORWEILER; YAKHOU, 2005).

Outro artigo de destaque é o de Hladchenko (2015), cujo objetivo foi uma análise comparativa dos BSC de quatro IES visando definir o quadro geral do BSC para uma instituição de ensino superior. Para a autora, o quadro apresentado no artigo pode ser utilizado como base para o desenvolvimento do quadro geral do Balanced Scorecard de outras IES.

Um outro trabalho relevante foi escrito e publicado em 2008 pelo finlandês Juha Kettunen. Teve como objetivo apresentar um quadro conceitual geral para ser usado na avaliação da qualidade do desempenho institucional no ensino superior. Kettunen (2008) ressalta que a gestão estratégica é amplamente utilizada em IES. Segundo ele, a abordagem do BSC foi concebida como um mecanismo para comunicar e implementar o plano estratégico e torná-lo mais compreensível para as partes interessadas.

Em linhas gerais, o referido estudo apresenta um modelo que é diretamente aplicável às universidades da Finlândia e pode ser modificado para as IES de outros países. O artigo mostra um mapa estratégico para as IES e apresenta os indicadores para as quatro perspectivas do BSC (KETTUNEN, 2008). 


\subsection{BSC EM INSTITUIÇÕES DE ENSINO FUNDAMENTAL E MÉDIO}

Esta seção analisa trabalhos com a aplicação do BSC em instituições de ensino fundamental e médio, foco do presente estudo. Observou-se uma escassez de trabalhos com BSC aplicados a instituições de ensino com este perfil, no entanto são descritos pontos relevantes de alguns artigos voltados para este foco.

O trabalho de Pereira e Melão (2012) traz uma investigação dos benefícios, obstáculos e desafios da implementação do BSC em escolas públicas fundamentais e de nível médio em um distrito de Portugal. Apesar das limitações, visto que o estudo tem o foco na realidade local, o mesmo é importante para mostrar o campo vasto de uso do BSC na educação fora do contexto do ensino superior, onde estão a maioria dos estudos, conforme enfatizado pelos próprios autores. Pereira e Melão (2012) ressaltam inclusive que a pesquisa deles foi uma das primeiras a discutir o uso do BSC em escolas públicas primárias e secundárias, e esperam que a mesma possa contribuir para a escassa literatura.

As etapas gerais executadas envolveram: definição da missão, visão e valores; análise da matriz SWOT; vetores e objetivos estratégicos; mapa estratégico; indicadores, metas e atividades; implementação; monitoramento e controle do projeto educativo e do plano anual de atividades. As propostas resultantes foram levadas à alta administração para aprovação. O plano anual das atividades foi concluído em dois meses, integrando as atividades desenvolvidas desde o início do ano letivo, de acordo com os objetivos definidos nas propostas. Os resultados mostram os objetivos, indicadores e metas para cada perspectiva (PEREIRA; MELÃO, 2012).

Num trabalho aplicado em escolas primárias e secundárias, Liang (2012), teve como base estudos anteriores sobre a aplicação do BSC aos sistemas de informação. Foram utilizados 21 indicadores de avaliação de desempenho para construir o modelo de pesquisa e desenvolver os itens do questionário baseado no modelo, com o objetivo de avaliar os departamentos de gerenciamento de sistemas de informação das escolas (LIANG, 2012).

Já o estudo de Tohidi et al. (2010), propõe um plano estratégico para instituições de ensino do Irã, especialmente escolas. As coletas de dados foram realizadas por entrevistas com gestores das suas instituições. O resultado foi um 
mapa estratégico com as relações de causa e efeito entre os objetivos de cada perspectiva do BSC. O mesmo é de considerável relevância para o presente trabalho, que desenvolve um mapa estratégico.

\section{METODOLOGIA}

O processo metodológico do presente trabalho inicia com o desenvolvimento de uma análise bibliométrica, a partir da pesquisa bibliográfica sobre BSC aplicado à gestão escolar, considerando as publicações entre os anos de 2005 a 2015. Procedeu-se então a coleta de vários artigos considerados mais relevantes, alguns deles comentados nas seções anteriores. Dentre eles foram selecionados alguns que trouxeram aplicação efetiva, seja com implantações, ou análises de Balanced Scorecards já efetivados em instituições de ensino. Destacam-se, entre estes artigos, abordagens com objetivos semelhantes à proposta da presente pesquisa, com desenvolvimento de modelos genéricos a partir de vários quadros de diferentes instituições, salientando que a proposta deste trabalho tem como foco propor um modelo para escolas fundamentais e de nível médio, que não é o foco da maioria dos trabalhos encontrados, voltados ao ensino superior. Com base nesses dados procedeu-se a montagem de um quadro preliminar de objetivos e indicadores para cada perspectiva do BSC.

Os quadros 1 a 4 contém as relações com os objetivos estratégicos e indicadores produzidas a partir da literatura, e foi utilizado nos questionários da presente pesquisa. São indicadas as fontes utilizadas, direta ou indiretamente, como base para a escolha desses objetivos. O quadro 1 diz respeito à perspectiva do aprendizado e crescimento. 
Quadro 1 - Objetivos Estratégicos - Perspectiva do Aprendizado e Crescimento

\begin{tabular}{|c|c|c|}
\hline Objetivos & Indicadores & Referências \\
\hline $\begin{array}{l}\text { 1. Melhorar as } \\
\text { habilidades em } \\
\text { Tecnologia da } \\
\text { Informação (TI) }\end{array}$ & $\begin{array}{c}\text { Número de treinamentos } \\
\text { anuais em recursos de TI; } \\
\text { Número de serviços e } \\
\text { tecnologias de Tl introduzidas } \\
\text { por ano. }\end{array}$ & $\begin{array}{c}\text { (TOHIDI; JAFARI; AFSHAR, } \\
\text { 2010);(PEREIRA; MELÃO, } \\
\text { 2012);(DORWEILER; YAKHOU, } \\
\text { 2005);(SCHOBEL; SCHOLEY, 2012) }\end{array}$ \\
\hline $\begin{array}{l}\text { 2. Priorizar as } \\
\text { inovações }\end{array}$ & $\begin{array}{l}\text { Quantidade de iniciativas } \\
\text { inovadoras por ano (Ex.: } \\
\text { Novas metodologias } \\
\text { pedagógicas) }\end{array}$ & $\begin{array}{l}\text { (SCHOBEL; SCHOLEY, 2012);(LIANG, } \\
\text { 2012);(PEREIRA; MELAO, } \\
\text { 2012);(DORWEILER; YAKHOU, 2005) }\end{array}$ \\
\hline $\begin{array}{l}\text { 3. Atrair e desenvolver } \\
\text { corpo docente de } \\
\text { qualidade }\end{array}$ & $\begin{array}{c}\text { Taxa de rotatividade do corpo } \\
\text { docente }\end{array}$ & $\begin{array}{c}\text { (SCHOBEL; SCHOLEY, } \\
\text { 2012);(DORWEILER; YAKHOU, } \\
\text { 2005);(KETTUNEN, 2008);(SAYED, } \\
\text { 2013) }\end{array}$ \\
\hline $\begin{array}{l}\text { 4. Atrair e desenvolver } \\
\text { uma equipe de } \\
\text { qualidade }\end{array}$ & $\begin{array}{l}\text { Taxa de rotatividade dos } \\
\text { funcionários }\end{array}$ & $\begin{array}{c}\text { (SCHOBEL; SCHOLEY, 2012);(SAYED, } \\
\text { 2013); (MORTLOCK, 2011) }\end{array}$ \\
\hline $\begin{array}{l}\text { 5. Melhorar a gestão } \\
\text { do conhecimento }\end{array}$ & $\begin{array}{c}\text { Número de iniciativas para } \\
\text { gerar e compartilhar } \\
\text { conhecimento }\end{array}$ & $\begin{array}{l}\text { (SCHOBEL; SCHOLEY, 2012); } \\
\text { (TSENG; FAN, 2011); (ZHAO; DE } \\
\text { PABLOS; QI, 2012) }\end{array}$ \\
\hline $\begin{array}{l}\text { 6. Fomentar a } \\
\text { cooperação entre } \\
\text { funcionários e } \\
\text { docentes }\end{array}$ & $\begin{array}{c}\text { Grau de cooperação entre } \\
\text { docentes e funcionários; Grau } \\
\text { de satisfação dos } \\
\text { colaboradores }\end{array}$ & $\begin{array}{c}\text { (SAYED, 2013);(OTIENO; AJOWI; DVC, } \\
\text { 2015); (ARUA, 2011) }\end{array}$ \\
\hline $\begin{array}{l}\text { 7. Apoiar o } \\
\text { desenvolvimento } \\
\text { cultural }\end{array}$ & $\begin{array}{c}\text { Número de iniciativas de } \\
\text { desenvolvimento cultural para } \\
\text { colaboradores (cursos, } \\
\text { seminários, eventos, etc.) } \\
\end{array}$ & $\begin{array}{c}\text { (TOHIDI; JAFARI; AFSHAR, } \\
\text { 2010);(LIANG, 2012);(PEREIRA; } \\
\text { MELÃO, 2012) }\end{array}$ \\
\hline $\begin{array}{l}\text { 8. Desenvolver } \\
\text { competências em } \\
\text { docentes e } \\
\text { funcionários }\end{array}$ & $\begin{array}{c}\text { Número de colaboradores } \\
\text { envolvidos em } \\
\text { desenvolvimento de } \\
\text { competências; Número médio } \\
\text { de horas de treinamento }\end{array}$ & $\begin{array}{c}\text { (LIANG, 2012);(PEREIRA; MELÃO, } \\
\text { 2012); (KETTUNEN, 2008) }\end{array}$ \\
\hline $\begin{array}{l}\text { 9. Alinhar funcionários } \\
\text { e docentes à } \\
\text { estratégia }\end{array}$ & $\begin{array}{l}\text { Grau de compromisso com a } \\
\text { estratégia da organização }\end{array}$ & $\begin{array}{l}\text { (LIANG, 2012);(DORWEILER; } \\
\text { YAKHOU, 2005); (MARR; NEELY, } \\
\text { 2003) }\end{array}$ \\
\hline
\end{tabular}

Fonte: Elaborado pelo autor

O Quadro 2 mostra as referências para os objetivos e indicadores da perspectiva dos processos internos do negócio. Estas informações, com exceção das referências, foram utilizadas no quadro correspondente no questionário. 
Quadro 2 - Objetivos Estratégicos - Perspectiva dos Processos Internos

\begin{tabular}{|c|c|c|}
\hline Objetivos & Indicadores & Referências \\
\hline $\begin{array}{l}\text { 1. Desenvolver gestão } \\
\text { de relacionamento } \\
\text { com clientes }\end{array}$ & $\begin{array}{c}\text { Satisfação dos clientes com os } \\
\text { canais de relacionamento; } \\
\text { Número de canais }\end{array}$ & $\begin{array}{l}\text { (TOHIDI; JAFARI; AFSHAR, 2010); } \\
\text { (RIZZO, 2009); (SOUZA, 2005); } \\
\text { (BETTENCOURT et al., 2015) }\end{array}$ \\
\hline $\begin{array}{l}\text { 2. Gerenciar } \\
\text { relacionamento com } \\
\text { fornecedores }\end{array}$ & $\begin{array}{l}\text { Eficiência no fornecimento dos } \\
\text { materiais e recursos } \\
\text { adquiridos junto aos } \\
\text { fornecedores }\end{array}$ & $\begin{array}{c}\text { (TOHIDI; JAFARI; AFSHAR, 2010); } \\
\text { (SOUZA, 2005); (BETTENCOURT et al., } \\
\text { 2015) }\end{array}$ \\
\hline $\begin{array}{l}\text { 3. Melhorar os } \\
\text { processos de TI }\end{array}$ & $\begin{array}{l}\text { Número de processos } \\
\text { informatizados; Número de } \\
\text { reclamações }\end{array}$ & $\begin{array}{c}\text { (TOHIDI; JAFARI; AFSHAR, 2010); } \\
\text { (MARCOS; ROUYET; BOSCH, 2011); } \\
\text { (ORTEGA; LORENCES; GÓMEZ, 2013) }\end{array}$ \\
\hline $\begin{array}{l}\text { 4. Gerenciar } \\
\text { dependência } \\
\text { (envolvimento) das } \\
\text { partes interessadas }\end{array}$ & $\begin{array}{l}\text { Número de reuniões de } \\
\text { planejamento com as partes } \\
\text { interessadas }\end{array}$ & $\begin{array}{l}\text { (SCHOBEL; SCHOLEY, 2012); } \\
\text { (PEREIRA; MELÃO, 2012); } \\
\text { (MABUSELA; DUMA, 2015) }\end{array}$ \\
\hline $\begin{array}{l}\text { 5. Desenvolver o } \\
\text { Ensino e } \\
\text { aprendizagem }\end{array}$ & $\begin{array}{l}\text { Satisfação dos professores, } \\
\text { alunos e demais envolvidos no } \\
\text { processo de ensino e } \\
\text { aprendizagem }\end{array}$ & $\begin{array}{l}\text { (SAYED, 2013);(DORWEILER; } \\
\text { YAKHOU, 2005); (BARMA; LACASSE; } \\
\text { MASSÉ-MORNEAU, 2014) }\end{array}$ \\
\hline $\begin{array}{l}\text { 6. Melhorar eficiência } \\
\text { operacional }\end{array}$ & $\begin{array}{l}\text { Tempo de resposta nos } \\
\text { Processos operacionais }\end{array}$ & $\begin{array}{l}\text { (SAYED, 2013);(DORWEILER; } \\
\text { YAKHOU, 2005); (PHILBIN, 2011) }\end{array}$ \\
\hline $\begin{array}{l}\text { 7. Garantir a } \\
\text { disponibilidade dos } \\
\text { recursos existentes }\end{array}$ & $\begin{array}{l}\text { Grau de reclamação quanto à } \\
\text { disponibilidade dos recursos } \\
\text { (salas, equipamentos, etc.) }\end{array}$ & $\begin{array}{c}\text { (SAYED, 2013); (PHILBIN, 2011); } \\
\text { (JOSEPH; YAKHOU; STONE, 2005) }\end{array}$ \\
\hline $\begin{array}{l}\text { 8. Melhorar a } \\
\text { satisfação de todos } \\
\text { com os processos }\end{array}$ & $\begin{array}{l}\text { Grau de satisfação das partes } \\
\text { interessadas com os } \\
\text { processos (professores, } \\
\text { funcionários, alunos) }\end{array}$ & $\begin{array}{l}\text { (SAYED, 2013); (LIANG, 2012); } \\
\text { (TSENG; FAN, 2011) }\end{array}$ \\
\hline 9. Gestão Institucional & $\begin{array}{l}\text { Grau de uso de recursos da } \\
\text { escola pelos alunos e } \\
\text { colaboradores; Taxa de } \\
\text { retenção de alunos; Taxa de } \\
\text { alunos concluintes } \\
\end{array}$ & $\begin{array}{c}\text { (SAYED, 2013);(PEREIRA; MELÃO, } \\
\text { 2012); (JOSEPH; YAKHOU; STONE, } \\
\text { 2005) }\end{array}$ \\
\hline $\begin{array}{l}\text { 10. Desenvolver o } \\
\text { marketing da escola }\end{array}$ & $\begin{array}{l}\text { Quantidade de atividades de } \\
\text { marketing desenvolvidas }\end{array}$ & $\begin{array}{l}\text { (TOHIDI; JAFARI; AFSHAR, 2010); } \\
\text { (MABUSELA; DUMA, 2015); } \\
\text { (BETTENCOURT et al., 2015) }\end{array}$ \\
\hline
\end{tabular}

Fonte: Elaborado pelo autor

O Quadro 3, por sua vez, contém os objetivos, indicadores e suas referências, dentro do contexto da perspectiva dos clientes. Analogamente às demais perspectivas, os objetivos e indicadores presentes no quadro foram apresentados no questionário para os gestores entrevistados. 
Quadro 3 - Objetivos Estratégicos - Perspectiva dos Clientes

\begin{tabular}{|c|c|c|}
\hline Objetivos & Indicadores & Referências \\
\hline $\begin{array}{l}\text { 1. Atração de novos } \\
\text { estudantes }\end{array}$ & $\begin{array}{l}\text { Quantidade de novos } \\
\text { estudantes por ano }\end{array}$ & $\begin{array}{l}\text { (SCHOBEL; SCHOLEY, 2012); } \\
\text { (MABUSELA; DUMA, 2015); } \\
\text { (HLADCHENKO, 2015) }\end{array}$ \\
\hline $\begin{array}{l}\text { 2. Manutenção dos } \\
\text { estudantes existentes }\end{array}$ & $\begin{array}{l}\text { Taxa de saída dos alunos } \\
\text { antes do último ano }\end{array}$ & $\begin{array}{c}\text { (SCHOBEL; SCHOLEY, 2012); (CHEN; } \\
\text { YANG; SHIAU, 2006); (HLADCHENKO, } \\
\text { 2015) }\end{array}$ \\
\hline $\begin{array}{l}\text { 3. Atender e superar } \\
\text { as expectativas de } \\
\text { serviço }\end{array}$ & $\begin{array}{c}\text { Percentual de estudantes } \\
\text { satisfeitos }\end{array}$ & $\begin{array}{c}\text { (TOHIDI; JAFARI; AFSHAR, 2010); } \\
\text { (LIANG, 2012); (KETTUNEN, 2008); } \\
\text { (DORWEILER; YAKHOU, } \\
\text { 2005);(SCHOBEL; SCHOLEY, 2012) }\end{array}$ \\
\hline $\begin{array}{l}\text { 4. Apoio para fomentar } \\
\text { programas } \\
\text { educacionais }\end{array}$ & $\begin{array}{l}\text { Número de contatos com } \\
\text { parceiros externos }\end{array}$ & $\begin{array}{c}\text { (PEREIRA; MELÃO, 2012); (SCHOBEL; } \\
\text { SCHOLEY, 2012); (PETRINI; } \\
\text { POZZEBON, 2009) }\end{array}$ \\
\hline $\begin{array}{l}\text { 5. Preparar os } \\
\text { estudantes para } \\
\text { ingressar em } \\
\text { universidades }\end{array}$ & $\begin{array}{l}\text { Percentual de egressos } \\
\text { aprovados em universidades } \\
\text { públicas e privadas }\end{array}$ & $\begin{array}{l}\text { (PEREIRA; MELÃO, 2012); } \\
\text { (MABUSELA; DUMA, 2015); } \\
\text { (THOMSEN, 2016) }\end{array}$ \\
\hline $\begin{array}{l}\text { 6. Investir na imagem } \\
\text { do corpo docente }\end{array}$ & $\begin{array}{c}\text { Índice de satisfação com o } \\
\text { corpo docente interna e } \\
\text { externamente }\end{array}$ & $\begin{array}{c}\text { (DORWEILER; YAKHOU, 2005); } \\
\text { (PHILBIN, 2011); (JOSEPH; YAKHOU; } \\
\text { STONE, 2005) }\end{array}$ \\
\hline $\begin{array}{l}\text { 7. Desenvolver novos } \\
\text { serviços }\end{array}$ & $\begin{array}{c}\text { Quantidade de serviços } \\
\text { prestados }\end{array}$ & $\begin{array}{c}\text { (TOHIDI; JAFARI; AFSHAR, 2010); } \\
\text { (ZANGOUEINEZHAD; MOSHABAKI, } \\
\text { 2011); (JOSEPH; YAKHOU; STONE, } \\
\text { 2005) }\end{array}$ \\
\hline $\begin{array}{l}\text { 8. Proporcionar bons } \\
\text { resultados dos alunos } \\
\text { nas disciplinas }\end{array}$ & $\begin{array}{l}\text { Resultado estudantil (Ex.: } \\
\text { Notas, taxa de aprovação) }\end{array}$ & $\begin{array}{l}\text { (PEREIRA; MELÃO, 2012); (SAYED, } \\
\text { 2013); (MONTECINOS et al., 2014) }\end{array}$ \\
\hline $\begin{array}{l}\text { 9. Desenvolver } \\
\text { habilidades nos } \\
\text { estudantes }\end{array}$ & $\begin{array}{l}\text { Quantidade de habilidades } \\
\text { proporcionadas aos } \\
\text { estudantes desenvolverem }\end{array}$ & $\begin{array}{c}\text { (DORWEILER; YAKHOU, } \\
\text { 2005);(SAYED, 2013); } \\
\text { (ZANGOUEINEZHAD; MOSHABAKI, } \\
\text { 2011) } \\
\end{array}$ \\
\hline $\begin{array}{l}\text { 10. Investir na gestão } \\
\text { da marca }\end{array}$ & $\begin{array}{l}\text { Grau de conhecimento do } \\
\text { nome (marca) da escola na } \\
\text { região }\end{array}$ & $\begin{array}{c}\text { (TOHIDI; JAFARI; AFSHAR, 2010); } \\
\text { (MARCOS; ROUYET; BOSCH, 2011); } \\
\text { (JEVONS et al., 2013) }\end{array}$ \\
\hline
\end{tabular}

Fonte: Elaborado pelo autor

Finalmente, o Quadro 4 mostra a relação entre objetivos e indicadores usados nos questionários da perspectiva financeira. Cada um deles com as suas respectivas referências. 
Quadro 4 - Objetivos Estratégicos - Perspectiva Financeira

\begin{tabular}{|c|c|c|}
\hline Objetivos & Indicadores & Referências \\
\hline $\begin{array}{l}\text { 1. Aumentar a } \\
\text { receita }\end{array}$ & $\begin{array}{l}\text { Receita total; Número de } \\
\text { inadimplentes }\end{array}$ & $\begin{array}{c}\text { (DORWEILER; YAKHOU, 2005); } \\
\text { (PEREIRA; MELÃO, 2012); } \\
\text { (PAPENHAUSEN; EINSTEIN, 2006) }\end{array}$ \\
\hline $\begin{array}{l}\text { 2. Aumentar a } \\
\text { receita total a partir } \\
\text { do número de alunos }\end{array}$ & $\begin{array}{c}\text { Quantidade total de alunos; } \\
\text { Número de novos estudantes; } \\
\text { Índice de evasão; Receita } \\
\text { prevista }\end{array}$ & $\begin{array}{l}\text { (KAPLAN; NORTON, 1996); } \\
\text { (PAPENHAUSEN; EINSTEIN, 2006); } \\
\text { (MABUSELA; DUMA, 2015) }\end{array}$ \\
\hline $\begin{array}{l}\text { 3. Diminuição do } \\
\text { custo total dos } \\
\text { serviços }\end{array}$ & $\begin{array}{l}\text { Custo mensal médio total dos } \\
\text { serviços; Receita líquida }\end{array}$ & $\begin{array}{l}\text { (TOHIDI; JAFARI; AFSHAR, 2010); } \\
\text { (YOUCHUN; JIANPENG, 2009); } \\
\text { (CHEN; YANG; SHIAU, 2006) }\end{array}$ \\
\hline $\begin{array}{l}\text { 4. Investir na } \\
\text { capacidade de } \mathrm{TI}\end{array}$ & $\begin{array}{l}\text { Custo decrescido com a } \\
\text { eficiência nos processos }\end{array}$ & $\begin{array}{l}\text { (HLADCHENKO, 2015); (CHEN; YANG; } \\
\text { SHIAU, 2006); (YOUCHUN; JIANPENG, } \\
\text { 2009) }\end{array}$ \\
\hline $\begin{array}{l}\text { 5. Melhorar o retorno } \\
\text { sobre o capital } \\
\text { empregado }\end{array}$ & $\begin{array}{l}\text { Taxa de retorno sobre o } \\
\text { investimento }\end{array}$ & $\begin{array}{c}\text { (TOHIDI; JAFARI; AFSHAR, 2010); } \\
\text { (REID, 2011); (RIBEIRO; GOMES, } \\
\text { 2009) }\end{array}$ \\
\hline $\begin{array}{l}\text { 6. Executar ações } \\
\text { para permitir } \\
\text { aumentar dos preços } \\
\text { dos serviços }\end{array}$ & Preços das mensalidades & $\begin{array}{c}\text { (TOHIDI; JAFARI; AFSHAR, 2010); } \\
\text { (KAPLAN; NORTON, 2000); (OTLEY, } \\
\text { 1999) }\end{array}$ \\
\hline $\begin{array}{l}\text { 7..Aumentar a } \\
\text { produtividade do } \\
\text { capital humano }\end{array}$ & $\begin{array}{c}\text { Relação funcionário/aluno; Valor } \\
\text { investido em gestão de recursos } \\
\text { humanos }\end{array}$ & $\begin{array}{c}\text { (DORWEILER; YAKHOU, 2005); } \\
\text { (ZANGOUEINEZHAD; MOSHABAKI, } \\
\text { 2011); (RUSKOV; TODOROVA, 2008) }\end{array}$ \\
\hline $\begin{array}{l}\text { 8. Aprimorar a } \\
\text { gestão dos recursos }\end{array}$ & $\begin{array}{l}\text { Índice de subutilização de } \\
\text { equipamentos, salas, etc. }\end{array}$ & $\begin{array}{c}\text { (DORWEILER; YAKHOU, 2005); } \\
\text { (HLADCHENKO, 2015); (PEREIRA; } \\
\text { MELÃO, 2012) }\end{array}$ \\
\hline $\begin{array}{l}\text { 9. Aumentar as } \\
\text { fontes de receita }\end{array}$ & $\begin{array}{c}\text { Quantidade (diversidade) das } \\
\text { fontes de receita; produtividade } \\
\text { das fontes }\end{array}$ & $\begin{array}{c}\text { (SAYED, 2013);(HLADCHENKO, 2015); } \\
\text { (REID, 2011) }\end{array}$ \\
\hline
\end{tabular}

Fonte: Elaborado pelo autor

\subsection{Critérios para a aplicação dos questionários}

Os critérios utilizados para escolha das escolas participantes, e posterior aplicação dos questionários, foram os seguintes:

Escolas privadas de ensino fundamental e nível médio com pelo menos 100 alunos. As instituições são localizadas no município do Natal, capital do estado do Rio Grande do Norte, bem como em seus municípios limítrofes, Parnamirim e São Gonçalo do Amarante.

A relação de todas as escolas com esses parâmetros foi obtida através da página Data Escola Brasil do portal do INEP, Instituto Nacional de Estudos e Pesquisas Educacionais Anísio Teixeira, autarquia federal vinculada ao Ministério da Educação (MEC) do Brasil. Também foram obtidas informações junto à prefeitura de Natal. A partir dessas fontes, foi preparada a lista das instituições, com endereços, telefones e quantidade de alunos de cada uma delas. 
Com base na relação de escolas, foi realizado um sorteio aleatório para a escolha das instituições onde seriam aplicados os questionários. Para isso, as instituições foram divididas em quatro grupos (extratos), de acordo com o número de alunos, de modo a obter-se uma amostra de questionários com participação efetiva de instituições de diferentes tamanhos. Os sorteios foram feitos alternadamente e equitativamente entre os grupos.

Os grupos foram divididos de acordo com os seguintes quantitativos: 100 a 350; 351 a 699; 700 a 999; 1000 ou mais alunos. A escolha desses parâmetros foi feita de modo que houvesse um equilíbrio no número de escolas em cada grupo. Os dois primeiros grupos ficaram com dezesseis escolas, enquanto os dois últimos foram compostos por quinze escolas. A amostra foi dividida proporcionalmente entre os quatro extratos.

O universo de escolas no sorteio foi 62, sendo 49 de Natal, doze de Parnamirim, e uma de São Gonçalo do Amarante, incluindo a totalidade de escolas da região, dentro do perfil escolhido. Algumas instituições possuem múltiplas unidades sob a mesma administração, porém, cada uma delas foi considerada individualmente, visto que em geral todas possuem diretores ou gerentes e são relacionadas separadamente no site do INEP. Foi realizada uma entrevista por escola, de modo que cada uma tivesse o mesmo peso nos resultados. Os questionários foram aplicados em 32 escolas, sendo, portanto, este o tamanho da amostra.

A amostragem foi, portanto, probabilística, pois todos os elementos (escolas) tiveram uma probabilidade diferente de zero de pertencerem à amostra, e a escolha foi feita através de sorteio.

O passo seguinte do processo foi a aplicação dos questionários através de entrevistas. Estas entrevistas foram feitas preferencialmente com os diretores ou, em caso de impossibilidade, com gerentes administrativos ou coordenadores das escolas. A escolha de entrevistas como técnica de pesquisa se deu para que houvesse uma melhor compreensão por parte dos gestores sobre as questões, bem como facilitar ao entrevistador sanar as eventuais dúvidas. Corroborando com este ponto de um melhor entendimento, Machado (2013) também usou a técnica de entrevistas em seu trabalho sobre BSC aplicado com empresas portuguesas, enquanto Pereira Filho et al. (2017) também aplicaram questionários a partir de Revista Produção Online. Florianópolis, SC, v. 18, n. 4, p. 1181-1205, 2018. 
entrevistas com gestores e, neste caso também com discentes de uma IES para aferir o nível de serviço da instituição.

Para a realização do sorteio, foi produzida uma planilha com a identificação das escolas, endereço, quantidade de alunos e um número para identificá-las. Uma função foi usada para gerar aleatoriamente um número correspondente a uma escola. Os sorteios foram feitos de acordo com os grupos de escolas conforme quantidade de alunos, como já mencionado.

Cada escola sorteada foi contatada para agendar a entrevista. Em alguns casos, dependendo da disponibilidade do entrevistado, os questionários foram enviados por e-mail após explicação prévia por telefone sobre o caráter e os objetivos da pesquisa. No total foram realizadas vinte entrevistas pessoalmente e doze à distância. No caso das escolas sorteadas nas quais não foi possível realizar a entrevista, seja pessoalmente ou mesmo à distância, foram sorteadas outras escolas para substituir as primeiras, mantendo a característica probabilística da amostra. Esses novos sorteios foram feitos respeitando os grupos.

\subsection{Detalhamentos dos Questionários}

Os questionários foram divididos em duas partes, sendo a primeira com perguntas para coletar dados como a identificação da escola, do entrevistado, a confirmação do número atualizado de alunos, além de informações sobre a gestão estratégica da instituição, com questões para identificar se a escola tem uma missão definida e propagada entre os funcionários, se possui objetivos estratégicos formalizados, se fazem algum tipo de medição de seu desempenho através de indicadores, se tem e como utilizam sistema de informação de gestão escolar e se consideram viável a implantação de um sistema de gestão estratégica.

A segunda parte do questionário foi considerada a principal da pesquisa, por ser a base para o desenvolvimento do quadro do BSC. Foi composta por questões objetivas para obter dos entrevistados quais são, de acordo com suas experiências, os principais objetivos estratégicos e indicadores para gestão escolar dentro de cada perspectiva do BSC. O conjunto de objetivos e indicadores apresentados nas questões foram exatamente os que estão presentes nos quadros 1 a 4 . Cada uma 
das quatro questões se referiu a uma perspectiva, de modo que o entrevistado deveria escolher no mínimo três e no máximo quatro itens em cada questão.

A estratégia utilizada foi baseada na técnica "bag of stars" (GARCIA; KLEIN, 2012). No caso da presente pesquisa, os objetivos mais votados, isto é, com a maior quantidade de "estrelas" recebidas, serviram como parâmetro para a produção do modelo do BSC. As "estrelas" foram tão somente a limitação dada aos entrevistados, na qual os mesmos deveriam marcar no mínimo três e no máximo quatro itens para cada perspectiva.

Em seguida, de acordo com a análise dos resultados dos questionários e observando-se os objetivos e medidas preferidos, vem a realização de um processo de geração de consenso para a definição de quatro objetivos com seus indicadores para cada perspectiva. Assim, elaborou-se um modelo de BSC que representa o perfil das escolas escolhidas. Esse processo foi descrito por Kaplan e Norton (1997) como uma das etapas na elaboração do BSC.

Para isso foram considerados primeiramente os quatro objetivos "mais votados" para cada quadro, e a partir deles foi feita uma análise se os mesmos abrangiam todos os aspectos das perspectivas. Estes aspectos, ou subperspectivas, foram baseados na teoria descrita por Kaplan e Norton (1997), e definidos da seguinte forma:

- Perspectiva do Aprendizado e Crescimento. Considerou-se as três categorias mencionadas por Kaplan e Norton (1996): 1) Capacidade dos funcionários; 2) Capacidade dos sistemas de informação; 3) Motivação, empowerment (descentralização de poderes ou grau de liberdade dos funcionários) e alinhamento com os objetivos da organização.

- Perspectiva do Processos Internos. Foi considerado o modelo da cadeia de valores genérica, também apresentado por Kaplan e Norton (1997): 1) Processo ou ciclo de inovação; 2) Processo ou ciclo de operações; 3) Processo ou ciclo de serviço pós-venda.

- Perspectiva do Clientes. Para esta visão foram consideradas as medidas essenciais apresentadas por Kaplan e Norton (1997), com exceção das medidas de lucratividade e participação no mercado, que são consequência direta das outras três e estão mais ligadas ao aspecto financeiro. Assim, as medidas consideradas foram: 1) Captação dos clientes, relacionada à qualidade de 
produtos e serviços; 2) Satisfação dos clientes, impactada pela imagem e reputação da instituição; 3) Retenção dos clientes, consequência do aspecto do relacionamento com os mesmos.

- Perspectiva Financeira. Para a área financeira considerou-se os três temas financeiros apresentados por Kaplan e Norton (1997): 1) Crescimento e mix de receitas; 2) Redução de custos / Aumento de produtividade; 3) Utilização de ativos / Estratégia de investimentos.

Portanto, conforme ressaltado, os quatro objetivos mais votados nos questionários não foram diretamente considerados para o quadro final do BSC. Antes disso, foi avaliado se as subperspectivas mencionadas foram contempladas com esses objetivos. Em caso negativo, o(s) objetivo(s) com a menor quantidades de votos (estrelas) foram substituídos por outro(s) por ordem de votação e que tratassem de aspectos não abarcados inicialmente.

O último processo tratou do desenvolvimento de um mapa estratégico a partir do modelo desenvolvido. $O$ objetivo foi dar subsídios para uma futura implantação do BSC por parte das escolas, podendo-se utilizar o mapa, juntamente com o modelo, como base, adaptando-os à realidade de cada instituição. O mapa foi montado a partir da análise das relações de causa e efeito entre os objetivos, fundamentando-se nas referências citadas.

\section{RESULTADOS}

Os resultados da pesquisa, conforme descrito nos objetivos, resumem-se em três principais. Primeiramente, a apresentação do panorama geral das escolas pesquisadas, no que diz respeito principalmente à gestão estratégica destas instituições. O segundo resultado foi o modelo do BSC para escolas com ensino fundamental e médio, dentro do perfil das instituições pesquisadas. $O$ terceiro resultado importante foi a obtenção, a partir do quadro e com base fundamentada na literatura, de um mapa estratégico para as referidas instituições. 


\subsection{Panorama geral da gestão estratégica das escolas}

Em relação à gestão estratégica, as escolas pesquisadas possuíam, em regra, missões definidas, mas as mesmas não eram propagadas adequadamente, salvo exceções. De modo geral, as escolas possuíam objetivos estratégicos, mas eles não eram formalizados. A medição de desempenho, na maioria dos casos, restringe-se aos aspectos de desempenho escolar.

Todas as escolas participantes possuíam software de gestão escolar, sendo alguns deles mais completos. Mas de um modo geral, de acordo com os gestores, os sistemas poderiam ser melhor utilizados, através do uso das informações para a gestão. A maioria das instituições não usa os softwares como ferramenta de gestão estratégica, limitando-se muitas vezes a aspectos de controle de resultados estudantis. Finalizando, sobre ferramentas para gestão estratégica, como o BSC, todos os entrevistados consideram viável a implementação.

\subsection{Definição do Modelo do Balanced Scorecard}

A seguir é mostrada a definição dos quatro objetivos estratégicos de cada perspectiva para compor o modelo do BSC, um dos principais resultados deste trabalho. Os objetivos e medidas da perspectiva do Aprendizado e Crescimento, escolhidos para compor o modelo, foram determinados de acordo com os resultados presentes na tabela 1 e com os aspectos considerados, que foram: 1) Capacidade dos sistemas de informação; 2) Capacidade dos funcionários; 3) Motivação, empowerment e alinhamento com os objetivos da organização. Esta numeração que identifica os aspectos, ou subperspectivas, foi usada nas tabelas de 1 a 4 , isso também será observado nas próximas seções para as demais perspectivas. Os quatro objetivos mais votados relativos ao Aprendizado e Crescimento (tabela 1) englobaram os três aspectos considerados e foram escolhidos para o modelo do BSC. Esses objetivos foram: Melhorar a gestão do conhecimento; desenvolver competências em docentes e funcionários; apoiar o desenvolvimento cultural; e fomentar a cooperação entre funcionários e docentes.

Para a perspectiva dos processos internos, os aspectos indicados foram: 1) Processo ou ciclo de inovação; 2) Processo ou ciclo de operações; 3) Processo ou 
ciclo de serviço pós-venda. A partir destas subperspectivas e dos resultados do questionário (tabela 2), dos cinco objetivos preferidos pelos gestores entrevistados, os quatro escolhidos foram: Desenvolver gestão de relacionamento com clientes; desenvolver o Ensino e aprendizagem; gerenciar dependência das partes interessadas; e melhorar os processos de TI. Este último teve o mesmo número de votos do objetivo "Melhorar a satisfação de todos com os processos", mas foi escolhido por estar ligado ao ciclo de inovação, não abrangido pelos outros três objetivos.

Tabela 1 - Resultados dos questionários da perspectiva do Aprendizado e Crescimento

\begin{tabular}{ccc}
\hline Objetivo & Votos & Aspectos \\
\hline $\begin{array}{c}\text { Melhorar a gestão do } \\
\text { conhecimento } \\
\quad \text { Apoiar o }\end{array}$ & 24 & 1 \\
$\begin{array}{c}\text { desenvolvimento cultural } \\
\text { Desenvolver } \\
\text { competências em }\end{array}$ & 17 & 3 \\
$\begin{array}{c}\text { docentes e funcionários } \\
\text { Fomentar a cooperação } \\
\text { entre funcionários e } \\
\quad \text { docentes }\end{array}$ & 12 & 3 \\
$\begin{array}{c}\text { Atrair e desenvolver } \\
\text { corpo docente de } \\
\text { qualidade }\end{array}$ & 11 & 2 \\
$\begin{array}{c}\text { Atrair e desenvolver uma } \\
\text { equipe de qualidade }\end{array}$ & 11 & 2 \\
$\begin{array}{c}\text { Priorizar as inovações } \\
\text { Melhorar as habilidades } \\
\text { em Tecnologia da } \\
\text { Informação }\end{array}$ & 10 & 1 \\
$\begin{array}{c}\text { Alinhar funcionários e } \\
\text { docentes à estratégia }\end{array}$ & 7 & 3 \\
\hline
\end{tabular}

Fonte: Elaboração própria
Tabela 2 - Resultados dos questionários da perspectiva dos Processos Internos

\begin{tabular}{ccc}
\hline Objetivo & Votos & Aspectos \\
\hline $\begin{array}{c}\text { Desenvolver Gestão de } \\
\text { relacionamento com } \\
\text { clientes }\end{array}$ & 22 & 3 \\
$\begin{array}{c}\text { Desenvolver o Ensino e } \\
\text { Aprendizagem }\end{array}$ & 20 & 2 \\
$\begin{array}{c}\text { Gerenciar dependência } \\
\text { das partes interessadas } \\
\text { Melhorar os processos } \\
\text { de TI }\end{array}$ & 17 & 2 \\
$\begin{array}{c}\text { Melhorar a satisfação de } \\
\text { todos com os processos } \\
\text { Desenvolver o }\end{array}$ & 13 & 2 e 3 \\
marketing da escola \\
Melhorar eficiência \\
operacional
\end{tabular}

Para a perspectiva dos clientes, foram consideradas as medidas essenciais indicadas no capítulo 3: 1) Captação dos clientes; 2) Satisfação dos clientes; 3) Retenção dos clientes. Estes aspectos devem estar contemplados pelos objetivos selecionados.

Apesar de outros aspectos importantes, definiu-se para o modelo os quatro objetivos preferidos pelos gestores, conforme tabela 3, que englobam as medidas de captação, retenção e satisfação dos clientes. Assim, os objetivos escolhidos para a 
perspectivas são: Desenvolver habilidades nos estudantes; preparar os estudantes para ingressar em universidades; proporcionar bons resultados dos alunos nas disciplinas; e atrair novos estudantes.

Para a perspectiva financeira, os temas citados por Kaplan e Norton (1996) e considerados para o modelo são: 1) Crescimento e mix de receitas; 2) Redução de custos / Aumento de produtividade; 3) Utilização de ativos / estratégia de investimentos.

Os quatro objetivos mais votados pelos gestores, conforme tabela 4, englobam apenas dois aspectos, ficando a redução de custos descoberta. Assim, substituímos no nosso modelo final o quarto item mais votado, aumento da receita, pelo quinto, diminuição do custo total do serviço, concluindo a escolha dos objetivos da perspectiva. Assim, os objetivos financeiros do modelo são: Aumentar a receita total a partir do número de alunos; aumentar a produtividade do capital humano; aprimorar a gestão dos recursos; e diminuição do custo total do serviço.

Tabela 3 - Resultados dos questionários dos objetivos da perspectiva dos clientes

\begin{tabular}{|c|c|c|}
\hline № do Objetivo & Votos & Aspectos \\
\hline $\begin{array}{c}\text { Desenvolver habilidades } \\
\text { nos estudantes }\end{array}$ & 20 & 3 \\
\hline $\begin{array}{l}\text { Preparar os estudantes } \\
\text { para ingressar em } \\
\text { universidades }\end{array}$ & 19 & 2 \\
\hline $\begin{array}{l}\text { Proporcionar bons } \\
\text { resultados dos alunos } \\
\text { nas disciplinas }\end{array}$ & 17 & 2 \\
\hline $\begin{array}{l}\text { Atração de novos } \\
\text { estudantes }\end{array}$ & 17 & 1 \\
\hline $\begin{array}{c}\text { Manutenção dos } \\
\text { estudantes existentes }\end{array}$ & 15 & 3 \\
\hline $\begin{array}{l}\text { Atender e superar as } \\
\text { expectativas do serviço }\end{array}$ & 14 & 2 \\
\hline $\begin{array}{l}\text { Investir na imagem do } \\
\text { corpo docente }\end{array}$ & 8 & 1 \\
\hline $\begin{array}{c}\text { Investir na gestão da } \\
\text { marca }\end{array}$ & 6 & 1 \\
\hline $\begin{array}{c}\text { Apoio para fomentar } \\
\text { programas educacionais }\end{array}$ & 5 & 3 \\
\hline $\begin{array}{c}\text { Desenvolver novos } \\
\text { serviços }\end{array}$ & 3 & 1 e 3 \\
\hline
\end{tabular}

Tabela 4 - Resultados dos questionários dos objetivos da perspectiva financeira

\begin{tabular}{|c|c|c|}
\hline № do Objetivo & Votos & Aspectos \\
\hline $\begin{array}{c}\text { Aumentar a receita total } \\
\text { a partir do número de } \\
\text { alunos }\end{array}$ & 23 & 1 \\
\hline $\begin{array}{c}\text { Aumentar a } \\
\text { produtividade do capital } \\
\text { humano }\end{array}$ & 20 & 3 \\
\hline $\begin{array}{c}\text { Aprimorar a gestão dos } \\
\text { recursos }\end{array}$ & 18 & 3 \\
\hline Aumentar a receita & 14 & 1 \\
\hline $\begin{array}{l}\text { Diminuição do custo } \\
\text { total do serviço }\end{array}$ & 11 & 2 \\
\hline $\begin{array}{l}\text { Melhorar o retorno } \\
\text { sobre o capital }\end{array}$ & 10 & 3 \\
\hline $\begin{array}{c}\text { Investir na capacidade } \\
\text { de TI }\end{array}$ & 7 & 2 \\
\hline $\begin{array}{c}\text { Aumentar as fontes de } \\
\text { receita }\end{array}$ & 6 & 1 \\
\hline $\begin{array}{l}\text { Executar ações para } \\
\text { permitir aumentar dos } \\
\text { preços dos serviços }\end{array}$ & 5 & 1 \\
\hline
\end{tabular}




\subsection{Apresentação do Modelo Finalizado}

A figura 1 apresenta o modelo do BSC obtido, considerando os objetivos e indicadores de acordo com os critérios descritos. Foram considerados quatro objetivos para cada visão. Em alguns casos foram inseridos múltiplos indicadores, com base nas referências analisadas.

Figura 1 - Balanced Scorecard para Instituições de Ensino Privadas de Nível Fundamental e Médio

\begin{tabular}{|c|c|c|c|}
\hline \multicolumn{2}{|c|}{ Perspectiva Financeira } & \multicolumn{2}{|c|}{ Perspectiva dos Clientes } \\
\hline Objetivos & Indicadores & Objetives & Indicadores \\
\hline Dimimuir o custo total dos serviços & $\begin{array}{l}\text { Casto meassal médao total dos } \\
\text { servigos, Receita liquida }\end{array}$ & Atrair novos esfudantes & $\begin{array}{l}\text { Quanitidade de novos estudantes por } \\
\text { ano }\end{array}$ \\
\hline $\begin{array}{l}\text { Aumentar a receita total a partir do } \\
\text { numero de aluaos }\end{array}$ & $\begin{array}{c}\text { Quastidade total de ahusos; Quant. de } \\
\text { novos estudautes, fnifice de evastos, } \\
\text { Receita prevista }\end{array}$ & $\begin{array}{l}\text { Propotcicour bons resultados dor } \\
\text { alunos das diseiplinas }\end{array}$ & $\begin{array}{l}\text { Resultado estudantil (Ex Notas, taxa } \\
\text { de aprovaçâ) }\end{array}$ \\
\hline $\begin{array}{c}\text { Aumentar a produtividade do capital } \\
\text { humano }\end{array}$ & $\begin{array}{l}\text { Relaçdo funcionairio alsmo: Valor } \\
\text { imvestido em gestito de recussios } \\
\text { humanos }\end{array}$ & $\begin{array}{l}\text { Preparar os estudantes para ingressat } \\
\text { en tuiversidades. }\end{array}$ & $\begin{array}{l}\text { Percentual de egressos aprovados em } \\
\text { vaiversidades póblicas e privadss }\end{array}$ \\
\hline Aprimotar a gestão dos recursos & $\begin{array}{l}\text { Indice de stabutilizxçato de } \\
\text { equipamentossalas, etc }\end{array}$ & $\begin{array}{l}\text { Desenvolver habilidides nos } \\
\text { estudantes }\end{array}$ & $\begin{array}{l}\text { Quantidade de habalidades } \\
\text { proposcionadas aos estudantes } \\
\text { desenvolverema }\end{array}$ \\
\hline \multicolumn{2}{|c|}{ Perspectiva dos Processos Internos do Negócio } & \multicolumn{2}{|c|}{ Perspectiva do aprendizado e crescimento } \\
\hline Objetivos & Indieadores & Objetives & Indicadores \\
\hline $\begin{array}{l}\text { Desenvolver pestilo de relacionamento } \\
\text { com clientes }\end{array}$ & $\begin{array}{l}\text { Satisfaçáo dos clieutes cous os canais de } \\
\text { relacionamento, Nümeno de canais }\end{array}$ & Melhorar al gevtio do conhosimento & 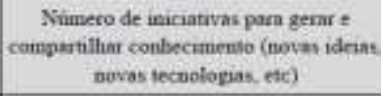 \\
\hline Melhonat os processon de TI & $\begin{array}{l}\text { Numero de processos informatizados; } \\
\text { Numero de reclamaçes }\end{array}$ & $\begin{array}{l}\text { Fomentar a cooperaçào entre } \\
\text { funcionários e dosentes }\end{array}$ & $\begin{array}{l}\text { Grau de cooperaçio entre docentes e } \\
\text { fimcioainios; Grau de tatisfaçio dos } \\
\text { colaborndores }\end{array}$ \\
\hline $\begin{array}{l}\text { Gerenciar dependencia des portes } \\
\text { interessadas: }\end{array}$ & $\begin{array}{c}\text { Nimero de reunióes de planejamento } \\
\text { com as partes interessadas }\end{array}$ & $\begin{array}{l}\text { Desenvolver competéncias em } \\
\text { docentes e funcionanos }\end{array}$ & $\begin{array}{c}\text { Nünero de colsboradores envolvidos no } \\
\text { devenvolvimento de competénciss; Horas } \\
\text { de trenamenso por pestoa }\end{array}$ \\
\hline Deseivolver o Ensino e aprendizagem & $\begin{array}{l}\text { Satisfacilo dos profersores, alunese } \\
\text { demuns emvolvidos no processo de ensano } \\
\text { e apreutizagean }\end{array}$ & Apoiar o desenvolvimento enltural & $\begin{array}{l}\text { Nuinero de mucutras de } \\
\text { desenvolvimento cultural (cursos. } \\
\text { keminifinc, eveatos ete) }\end{array}$ \\
\hline
\end{tabular}

Fonte: Elaboração própria

\subsection{Mapa estratégico}

O terceiro resultado deste trabalho foi a formulação de um mapa estratégico para as escolas com o perfil das instituições participantes desta pesquisa, a partir do quadro geral do BSC apresentado. O objetivo é mostrar a interação entre as perspectivas do BSC dentro do modelo desenvolvido, enfatizando as ligações entre os seus objetivos estratégicos. Ou seja, mostrar as relações de causa e efeito identificadas. Após análise dos objetivos de cada perspectiva, com base na literatura, chegamos a essas relações, que podem ser observadas no mapa estratégico desenvolvido, que está na figura 2. 
Figura 2 - Modelo de Mapa Estratégico para Instituições de Ensino Privadas de Nível Fundamental e Médio

\section{Perspectivas \\ Relações de causa e efeito entre os objetivos estratégicos}

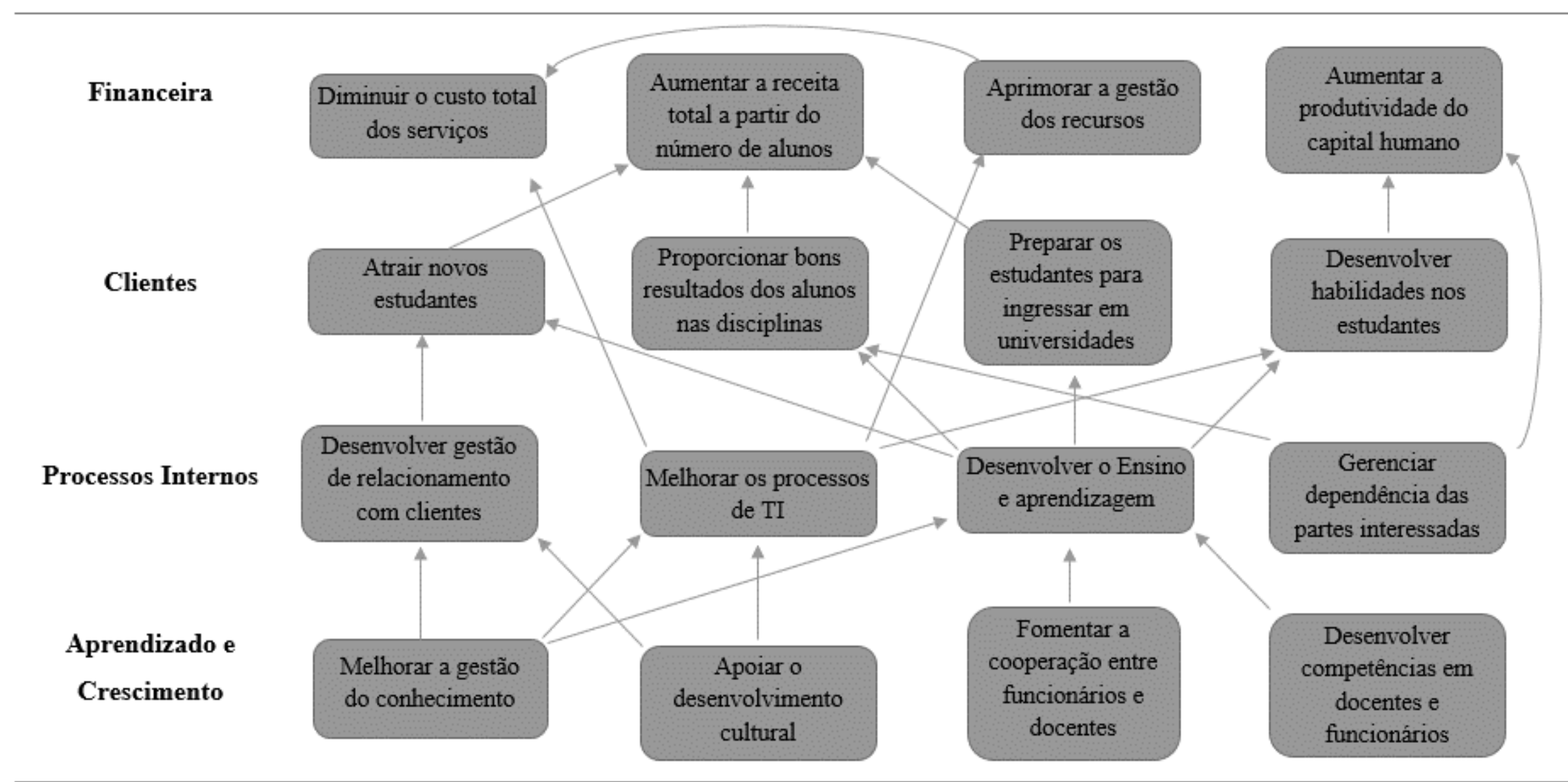

Fonte: Elaboração própria 


\section{CONSIDERAÇÕES FINAIS}

O BSC é uma metodologia para gestão estratégica consolidada mundialmente nas últimas décadas. A literatura mostra diversos trabalhos sobre o tema a cada ano, com aplicações nas mais diferentes áreas. Contudo, esta pesquisa mostrou uma escassez de estudos sobre o BSC na área educacional sobretudo associados ao ensino básico. A maioria das pesquisas identificadas nas principais bases de dados internacionais refere-se ao âmbito do ensino superior.

Dentro deste contexto, o presente trabalho propôs a aplicação do BSC na área de gestão educacional com o desenvolvimento de um modelo para instituições de ensino privadas, com foco nos níveis fundamental e médio, contribuindo assim com os estudos neste cenário. Os objetivos principais foram a descrição do panorama geral da gestão estratégica nas escolas pesquisadas e, principalmente, a elaboração de um modelo de BSC além do desenvolvimento de um mapa estratégico para as instituições, considerando o contexto mencionado.

Com a seleção, exploração e análise da literatura sobre o BSC, especialmente aplicado à área de educação, foi desenvolvido um modelo preliminar. A partir dele, elaborou-se um questionário com quadros para cada perspectiva, aprendizado e crescimento, processos internos, clientes e financeira, contendo objetivos e indicadores para cada uma delas. Para a aplicação dos questionários durante as entrevistas foi lançado mão da técnica "bag of stars", para filtrar os itens mais votados em cada perspectiva pelos gestores entrevistados.

A partir da análise dos dados dos questionários, considerando o quantitativo de votos para cada objetivo, bem como a cobertura dos diversos aspectos de cada perspectiva, foram definidos quatro objetivos com seus indicadores para cada uma delas, compondo-se o modelo final do BSC, aplicável às instituições com perfil semelhante ao das escolas pesquisadas. Com base no modelo e na literatura foi elaborado um mapa estratégico com as relações de causa e efeito entre os objetivos, a partir da análise da influência ou não de um objetivo para com os demais, partindo da perspectiva do aprendizado e crescimento, na parte inferior do mapa, até a perspectiva financeira, na parte superior.

O modelo elaborado é uma base para a aplicação do BSC em diferentes instituições de ensino, podendo ser adaptado à realidade de cada uma. O trabalho é uma referência para novos estudos sobre o tema, possibilitando a exploração da 
lacuna mencionada para aplicações do BSC na educação básica. Partindo do quadro com objetivos e indicadores, para colocar a gestão estratégica em ação, a instituição precisará definir metas e iniciativas indicando quando e como pretende alcançar os indicadores estipulados. O mapa estratégico elaborado contribuirá para o entendimento das relações de causa e efeito entre os objetivos, de modo que os gestores tenham uma maior compreensão dos relacionamentos entre os diversos setores e o impacto dos resultados de cada perspectiva sobre as demais, sendo também uma base para desenvolvimento de mapas dentro da área educacional.

\section{REFERÊNCIAS}

ARUA, Ushe. A Framework of Cooperation Between Academic Staff and Library Staff for a Meaningful University Education for Students Between Academic Staff and University Education for Students. Library Philosophy and Practice, 2011.

BARMA, Sylvie; LACASSE, Mathieu; MASSÉ-MORNEAU, Julie. Engaging discussion about climate change in a Quebec secondary school: A challenge for science teachers. Learning, Culture and Social Interaction, v. 4, p. 28-36, 2014. Disponível em: http://dx.doi.org/10.1016/j.Icsi.2014.07.004.

BETTENCOURT, Lance A. et al. Rethinking customer relationships. Business Horizons, v. 58, n. 1, p. 99-108, 2015. Disponível em: <http://dx.doi.org/10.1016/j.bushor.2014.09.003>.

CHEN, Shun-Hsing; YANG, Ching-Chow; SHIAU, Jiun-Yan. The application of balanced scorecard in the performance evaluation of higher education. The TQM Magazine, v. 18, $\mathrm{n}$. 2, p. 190-205, 2006.

DORWEILER, Vernon P.; YAKHOU, Mehenna. Scorecard for academic administration performance on the campus. Managerial Auditing Journal, v. 20, n. 2, p. 138-144, 2005.

GARCIA, Ana Cristina Bicharra; KLEIN, Mark. The Bag of Stars : High-Speed Idea Filtering for Open Innovation. MIT Sloan School of Management, n. september 2013, p. 1-14, 2012.

HLADCHENKO, Myroslava. Balanced Scorecard - a strategic management system of the higher education institution. International Journal of Educational Management, v. 29, n. 2, p. 167-176, 2015. Disponível em: http://www.emeraldinsight.com/doi/abs/10.1108/lJEM-112013-0164.

JEVONS, Colin et al. Introduction: Thought leadership in brand management. Journal of Business Research, v. 66, n. 1, p. 1-3, 2013.

JOSEPH, Mathew; YAKHOU, Mehenna; STONE, George. An educational institution's quest for service quality: customers' perspective. Quality Assurance in Education, v. 13, n. 1, p. 66-82, 2005.

KAPLAN, Robert S; NORTON, David P. A estratégia em ação: balanced scorecard. [S.I.]: Rio de Janeiro: Campus, 1997.

KAPLAN, Robert S; NORTON, David P. Linking the Balanced Scorecard to Strategy. 
California Management Review, v. 39, n. 1, p. 53-79, 1996.

KAPLAN, Robert S; NORTON, David R. Having Trouble with Your Strategy? Then Map It. Harvard Business Review, 2000.

KETTUNEN, Juha. A conceptual framework to help evaluate the quality of institutional performance. Quality Assurance in Education, v. 16, n. 4, p. 322-332, 2008.

LIANG, Yi-hui. Scorecard : a Case Study for the Elementary and Secondary. Proceedings of the 2012 International Conference on Machine Learning and Cybernetics, Xian, p. 1517, 2012.

MABUSELA, M S; DUMA, M A N. Implementing Practical Marketing Strategy for Township Schools. Anthropologist, v. 19, n. 3, p. 741-748, 2015.

MACHADO, Maria. Balanced Scorecard : um estudo empírico sobre pequenas e médias empresas Balanced Scorecard : an empirical study of small and medium size enterprises. RBGN - Revista Brasileira de Gestão de Negócios, p. 129-148, 2013.

MARCOS, Antonio Folgueras; ROUYET, Juan Ignacio; BOSCH, Antoni. An IT Balance Scorecard design under Service Management philosophy. Proceedings of the Annual Hawaii International Conference on System Sciences, p. 4972-4981, 2011.

MARR, Bernard; NEELY, Andy. Automating the balanced scorecard - selection criteria to identify appropriate software applications. Measuring Business Excellence, v. 7, n. 3, p. 29-36, 2003.

MONTECINOS, Carmen et al. A goal orientation analysis of teachers' motivations to participate in the school self-assessment processes of a quality assurance system in Chile. Educational Assessment, Evaluation and Accountability, p. 1-21, 2014.

MORTLOCK, Sue. A framework to develop leadership potential. Nursing Management, 2011.

ORTEGA, Marin; LORENCES, Pérez; GÓMEZ, Marx. Architecture for business intelligence design on the IT service management scope. Engineering and Management of IT-based Service Systems, 2013.

OTIENO, Kennedy Omondi; AJOWI, Jack Odongo; DVC, Joseph Bosire. Challenges Faced by the School Administration while Carrying out Various Welfare Practices on Secondary School Teachers in Bondo Sub-County, Kenya. Mediterranean Journal of Social Sciences, v. 6, n. 2, p. 479-488, 2015. Disponível em: http://www.mcser.org/journal/index.php/mjss/article/view/5833.

OTLEY, David. Performance management : a framework for management control systems research. Management Accounting Research, n. November 1998, p. 363-382, 1999.

PAPENHAUSEN, Chris; EINSTEIN, Walter. Implementing the Balanced Scorecard at a college of business. Measuring Business Excellence, v. 10, n. 3, p. 15-22, 2006.

PEREIRA, Maria Manuela; MELÃO, Nuno Filipe. The implementation of the balanced scorecard in a school district: Lessons learned from an action research study. International Journal of Productivity and Performance Management, v. 61, n. 8, p. 919-939, 2012.

PEREIRA FILHO, Evadio; et al. Em que atributos deve melhorar o nível de serviço? As 
concepções de gestores e alunos de uma IES privada. Produção Online, v. 17, n. 4, p. 1480-1498, 2017. DOI: http://dx.doi.org/10.14488/1676-1901.v17i4.2740.

PETRINI, Maira; POZZEBON, Marlei. Managing sustainability with the support of business intelligence: Integrating socio-environmental indicators and organisational context. Journal of Strategic Information Systems, v. 18, n. 4, p. 178-191, 2009. Disponível em: http://dx.doi.org/10.1016/j.jsis.2009.06.001.

PHILBIN, Simon P. Design and implementation of the Balanced Scorecard at a university institute. Measuring Business Excellence, v. 15, n. 3, p. 34-45, 2011.

QUESADO, Patrícia Rodrigues; GUZMAN, Beatriz Aibar;; RODRIGUES, Lúcia Lima. Determinant Factors of the Implementation of the Balanced Scorecard in Portugal : empirical evidence in public and private organizations. RBGN - Revista Brasileira de Gestão de Negócios, v. 16, n. 51, p. 199-222, 2014.

REID, Michele M. Is the balanced scorecard right for academic libraries? The Bottom Line: Managing Library Finances, v. 24, n. 2, p. 85-95, 2011.

RIBEIRO, Jorge; GOMES, R U I. IT Governance using COBIT implemented in a High Public Educational Institution - A Case Study 2 Information Technology Governance. Computing and Computational Intelligence, p. 41-52, 2009.

RIZZO, C. Gestão estratégica do aluno-cliente nas instituições de ensino : um estudo de caso. 2009. 2009.

RUSKOV, Petko; TODOROVA, Yanka. Learning and growth strategy metrics. Proceedings of the 9th International Conference on Computer Systems and Technologies and Workshop for PhD Students in Computing - CompSysTech '08, p. IIIB.1, 2008. Disponível em: http://portal.acm.org/citation.cfm?doid=1500879.1500920.

SAYED, Naqi. Ratify, reject or revise: balanced scorecard and universities. International Journal of Educational Management, v. 27, n. 3, p. 203-220, 2013. Disponível em: http://www.emeraldinsight.com/journals.htm?articleid=17066174\&show=abstract.

SCHOBEL, Kurt; SCHOLEY, Cam. Balanced Scorecards in education: focusing on financial strategies. Measuring Business Excellence, v. 16, n. 3, p. 17-28, 2012.

SOUZA, Fabiano Sales. Implementação do Balanced Scorecard ( BSC ) em uma organização do sistema s : o caso Senai / BA. Dissertação (mestrado) - Universidade Salvador - UNIFACS, Programa de Pós-Graduação em Administração, 2005., 2005.

THOMSEN, J.-P. Test-Based Admission to Selective Universities: A Lever for FirstGeneration Students or a Safety Net for the Professional Classes? Sociology, 2016.

TOHIDI, Hamid; JAFARI, Aida; AFSHAR, Aslan Azimi. Using balanced scorecard in educational organizations. Procedia - Social and Behavioral Sciences, v. 2, n. 2, p. 55445548, 2010. Disponível em:

http://www.sciencedirect.com/science/article/pii/S1877042810009444.

TSENG, Fan Chuan; FAN, Yen Jung. Exploring the Influence of Organizational Ethical Climate on Knowledge Management. Journal of Business Ethics, v. 101, n. 2, p. 325-342, 2011.

YOUCHUN, Tang; JIANPENG, Hu. Design of Management System for Computer 
laboratory Based on ITIL. p. 3519-3522, 2009.

ZANGOUEINEZHAD, Abouzar; MOSHABAKI, Asghar. Measuring university performance using a knowledge-based balanced scorecard. International Journal of Productivity and Performance Management, v. 60, n. 8, p. 824-843, 2011.

ZHAO, Jingyuan; DE PABLOS, Patricia Ordónez; QI, Zhongying. Enterprise knowledge management model based on China's practice and case study. Computers in Human Behavior, v. 28, n. 2, p. 324-330, 2012. Disponível em:

http://dx.doi.org/10.1016/j.chb.2011.10.001.

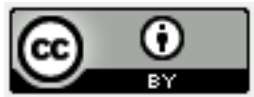

Artigo recebido em: 19/07/2017 e aceito para publicação em: 04/11/2018 DOI: http://dx.doi.org/10.14488/1676-1901.v18i4.2737 